\title{
Paradoxical Embolus Stuck in a Patent Foramen Ovale
}

\author{
Zaki Kohistani $^{1}$ Chris Probst ${ }^{1}$ \\ ${ }^{1}$ Department of Heart Surgery, University Clinics Bonn, Bonn, \\ Germany \\ Thorac Cardiovasc Surg Rep 2016;5:60-61.
}

\begin{abstract}
Address for correspondence Dr. med. Zaki Kohistani, Department of Heart Surgery, University Clinics Bonn, Sigmund-Freud-Straße 25, Bonn 53127, Germany (e-mail: zaki.kohistani@ukb.uni-bonn.de).
\end{abstract}

\begin{abstract}
In-hospital mortality rate of an embolus in transit is as high as $44.7 \%$. In some cases, a paradoxical embolus can get stuck in a patent foramen ovale. Because of the high mortality rate, this condition should be considered as an emergency case. Echocardiography has been established as the gold standard method for the diagnosis. A negative echocardiography, however, does not rule out an embolus in transit. To rule out pulmonary embolisms, a computed tomography scan of the chest should also be performed.

Keywords

- imaging

- pulmonary embolism

A cardiothoracic surgeon should be consulted immediately upon diagnosis of an embolus in transit. There is no medical consensus for the treatment of the above mentioned condition, however, surgical treatment appears to be the best approach in patients who are surgical candidates.
\end{abstract}

\section{Introduction}

A paradoxical embolism after deep vein thrombosis is a wellknown entity. If the foramen ovale is open, the embolus could cross to the arterial side and cause deadly ischemia. Visualization of the embolus passing the patent foramen ovale with echocardiography, computed tomography (CT) and intraoperative imaging is rare. In this case report we present the extraordinary diagnostic and surgical images of a paradoxical embolus stuck in a patent foramen ovale.

\section{Case Presentation}

A 56-year-old patient was admitted to the hospital with a diagnosed massive pulmonary embolism after deep vein thrombosis. A CT scan of the chest revealed multiple bilateral and central embolisms of the pulmonary arteries. Additionally, the echocardiography of the heart showed a large embolus in transit from the right atrium through an open foramen ovale into the left atrium and floating above the mitral valve ( - Fig. 1 ).

With the diagnosis of this large floating embolus into the left atrium and the mitral valve, the patient was taken as an emergency case into the operation room. During surgical exploration we found the large embolus stuck in the patent foramen ovale (-Fig. 2).

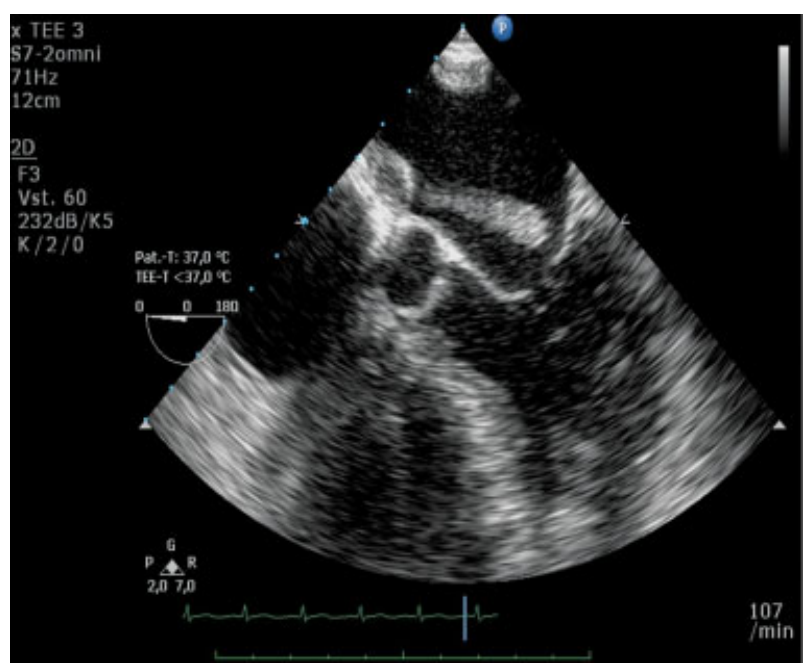

Fig. 1 Transesophageal image of the heart in apical 5 chamber view. The arrow shows the floating embolus in the left atrium, which has crossed the patent foramen ovale and hangs over the mitral valve. received

July 8,2016

accepted

August 5, 2016

published online

October 3, 2016
DOI http://dx.doi.org/

$10.1055 / \mathrm{s}-0036-1593395$. ISSN 2194-7635. (c) 2016 Georg Thieme Verlag KG Stuttgart · New York
License terms

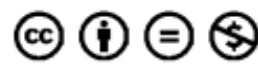




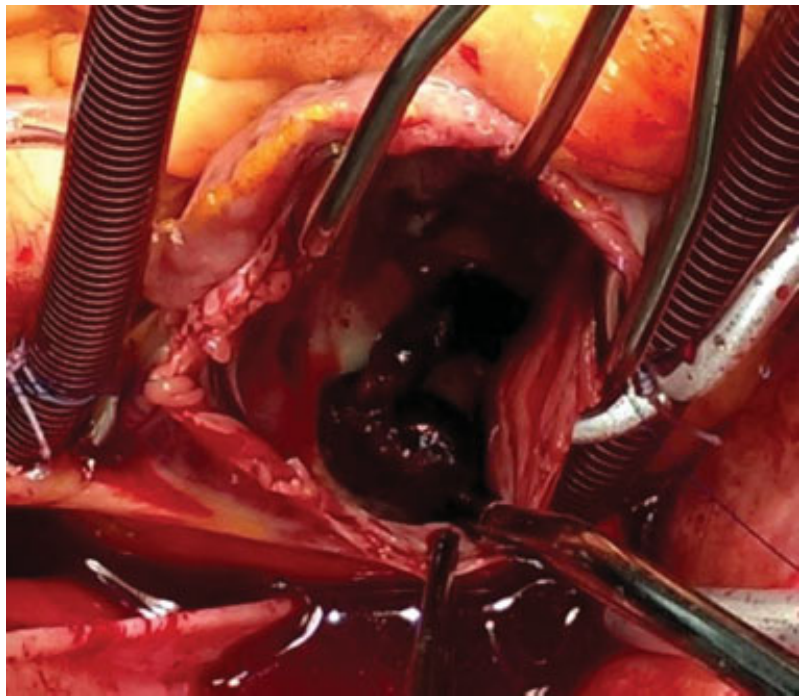

Fig. 2 Intraoperative image of the heart. The image shows the open right atrium with the paradoxical embolus (arrow) trying to cross the open foramen ovale in to the left atrium.

The embolus was completely extracted (-Fig. 3) and closure of the patent foramen ovale was performed. The multiple pulmonary embolisms could also be extracted (-Fig. 4). After an uneventful postoperative course, the patient was discharged.

\section{Discussion}

In-hospital mortality rate of an embolus in transit is as high as $44.7 \%{ }^{1}$ However, the prognosis is good after the embolus is

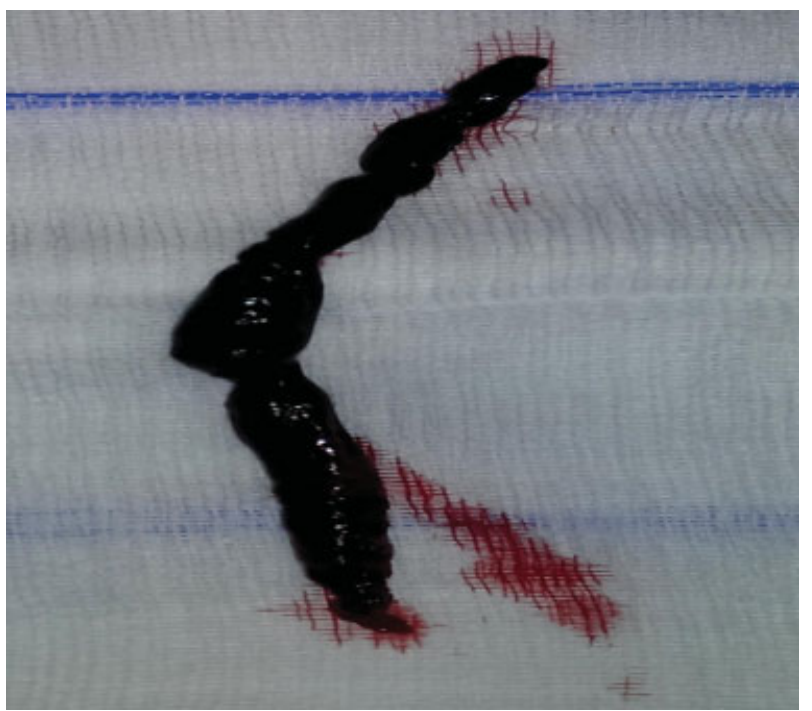

Fig. 3 The image shows the paradoxical embolus after the surgical extraction.

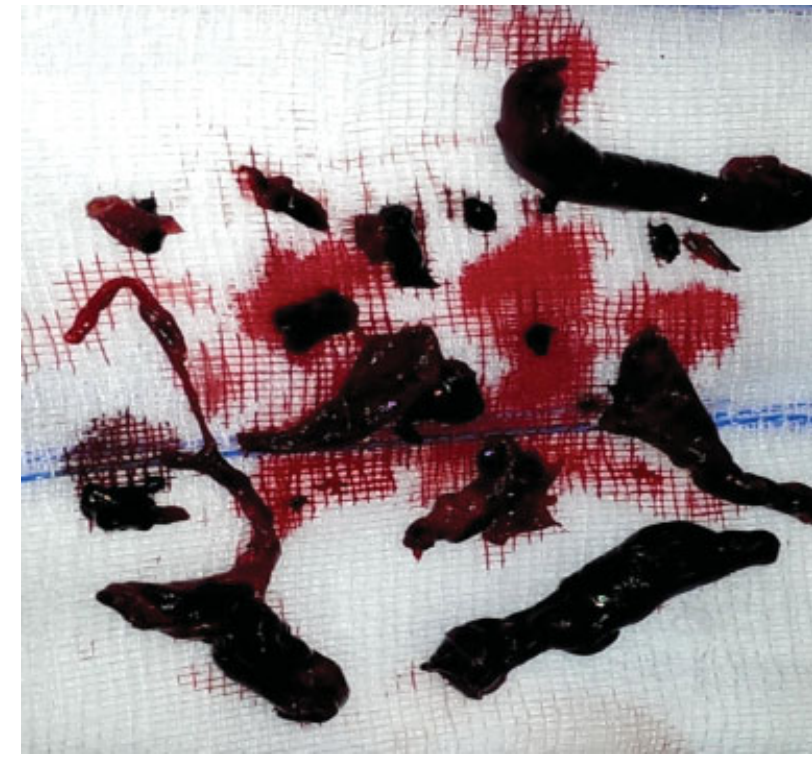

Fig. 4 The image shows multiple extracted lung embolisms.

extracted, closure of the patent foramen ovale is performed and an anticoagulation therapy is conducted. ${ }^{1}$ Echocardiography is the gold standard for the diagnosis of an embolus in transit. A negative echocardiography, however, does not rule out the above mentioned condition. ${ }^{2}$ To rule out pulmonary embolism, a CT scan of the chest is also essential.

Because of the high mortality rate, a surgical treatment should be considered as soon as an embolus in transit is diagnosed. ${ }^{3}$ There is no medical consensus about the best option for the treatment of an embolus in transit. However, compared with anticoagulation and thrombolysis, surgery is associated with fewer complications of recurrent paradoxical embolisms ${ }^{4}$ and appears to be the best approach in patients who are surgical candidates.

\section{References}

1 Chartier L, Béra J, Delomez M, et al. Free-floating thrombi in the right heart: diagnosis, management, and prognostic indexes in 38 consecutive patients. Circulation 1999;99(21):2779-2783

2 Pinto FJ. When and how to diagnose patent foramen ovale. Heart 2005;91(4):438-440

3 Podroužková H, Horváth V, Hlinomaz O, et al. Embolus entrapped in patent foramen ovale: impending paradoxical embolism. Ann Thorac Surg 2014;98(6):e151-e152

4 Fauveau E, Cohen A, Bonnet N, Gacem K, Lardoux H. Surgical or medical treatment for thrombus straddling the patent foramen ovale: impending paradoxical embolism? Report of four clinical cases and literature review. Arch Cardiovasc Dis 2008;101(10): 637-644 